\title{
Proceeding
}

Supplementary Issue: Spring Conferences of Sports Science. Costa Blanca Sports Science Events, 19-20 June 2020. Alicante, Spain.

\section{Motion analysis in the elderly: Evaluation of an APA program on the gait of elderly using an inertial motion capture system}

\author{
SILVIA COPPOLA , RODOLFO VASTOLA \\ Motion Analysis Laboratory, Department of Human, Philosophical and Educational Sciences, University of \\ Salerno, Italy
}

\begin{abstract}
Elderly walking performance undergoes a natural physiological decline that, from a biomechanical point of view, it is manifested by an alteration of the spatial-temporal characteristics of the gait. The quantitative assessment of the gait in the elderly requires an objective and reliable evaluation system that can be used under conditions of daily living. The aim of this study was to investigate, using an inertial motion capture system, the effects of five months of a structured Adapted Physical Activity (APA) program on the main characteristics of the gait in the elderly, detected directly within the homecare. Data relating to spatialtemporal parameters have been objectively and systematically acquired, through this wireless inertial sensor positioned on the L5 spinal segment, before and after the implementation of the APA program. The research was carried out in two Italian elderly centres. The sample consisted of 11 elderly aged between 70 and 92 years $(82.55 \pm 7.43)$. The results obtained found a substantial improvement in speed, cadence, stride length, symmetry index, cycle time, single support phase and swing duration after the implementation of the APA program. In conclusion, this study revealed a high feasibility of the APA structured program and a readable efficacy in the use of inertial motion capture system for the evaluation of the gait in the elderly homecare. In relation to the number of the sample, the results are more suggestive than conclusive, hence suggest the need for further research on the effects across a larger range of subjects.
\end{abstract}

Keywords: Gait; Elderly; Inertial motion capture systems; Motion analysis; Adapted Physical Activity.

\section{Cite this article as:}

Coppola, S., \& Vastola, R. (2020). Motion analysis in the elderly: Evaluation of an APA program on the gait of elderly using an inertial motion capture system. Journal of Human Sport and Exercise, 15(3proc), S664-S675. doi:https://doi.org/10.14198//hse.2020.15.Proc3.19

\footnotetext{
Corresponding author. Motion Analysis Laboratory, Department of Human, Philosophical and Educational Sciences, University of Salerno, Italy. https://orcid.org/0000-0003-4924-6864

E-mail: sicoppola@unisa.it

Supplementary Issue: Spring Conferences of Sports Science. Costa Blanca Sports Science Events, 19-20 June 2020. Alicante, Spain.

JOURNAL OF HUMAN SPORT \& EXERCISE ISSN 1988-5202

(C) Faculty of Education. University of Alicante

doi:10.14198/jhse.2020.15.Proc3.19
} 


\section{INTRODUCTION}

Walking performance involves different bio-mechanical and neurological components, including mobility of the joints of the lower limb (Baker et al., 2016), appropriate timing of muscle contraction as intramuscular and intermuscular coordination (Whittle, 2014), adequate intensity of muscle contraction (Williams \& Vicinanza, 2018) and efficient sensory input including vision, proprioception and vestibular system (Iwasaki \& Yamasoba, 2015). Decrease in visual acuity and sensitivity to contrasts are common in elderly (Iwata \& Kitamoto, 2020). The vestibular system decreases its efficiency, resulting in a decreased ability to detect signals related to the position and movements of the head (Goble et al., 2009). The decline of proprioceptive systems, involving neuromuscular spindles, Golgi tendon organ and corpuscle of Pacini, causes a reduced tactile sensitivity of the sole of the foot and reduces the afference of the signals coming from the centre of pressure (Jahn, 2019; Bizovska et al., 2015). Decrease in efficiency of receptors in muscle, tendon and joint, that involves neuromuscular spindles and Golgi tendon organs, leads to a decrease in the combination and integration of afferents related to positions and movements from different parts of the body as the relationship between limbs-trunk, trunk-head and arms-legs (Pirker \& Katzenschlager, 2017).

In scientific literature there are many studies that have investigated the physiological changes of gait in the elderly (Auvinet et al., 2017; Hamacheret al., 2011). Generally, these changes occur from the age of 65 (Cruz, 2017). There is a reduction of pace, characterized by a greater rigidity and control, a greater variability in the length and width of the step (Osoba et al., 2019), a wider lateral oscillations of the body with widening of the walking base, a reduction of joint excursion at the level of the lower limbs (Aboutorabi et al., 2016) that determines a less flexion of the knee, elevation of the heel at the beginning of the swing, less dorsal flexion of the foot in the final phase of the swing, an increase in time during the foot resting phase with a corresponding reduction in swing time resulting in a reduction of time in single-podalic support (Fan et al., 2016; Jahn et al., 2015). Gait in elderly is also influenced by the muscle strength deficiency and in particular by the muscle atrophy and sarcopenia, due to loss of motor neurons, muscle fibres and aerobic capacity (Morley, 2016). Other age-related changes may occur in the joints and can limit movement; the loss of passive range of motion in the elderly is often progressive and subtle (Miljkovic et al., 2015).

All these aspects related to the natural physiological decline that occurs in elderly can be countered by a specifically adapted physical activity program (Halaweh et al., 2015; Warburton \& Bredin, 2016) able to counteract the main factors that determine the decline in gait skills in the elderly (Lee \& Lee, 2017). The objectives of the programming of an adapted physical activity (APA) for elderly, in fact, foresee the improvement, the maintenance and the global recovery of the residual potential of the elderly (De Souto Barreto et al., 2016), with particular reference to the improvement of the ability of static and dynamic balance, the increase in muscle strength, the improvement of joint mobility and the function and coordination of the step (Coppola, 2018).

Dynamic balance and walking performance are skills characterized by a high plastic-vicariant component that, if properly stimulated and trained, through a specific program of physical activity, aimed at maintenance and improving the motor prerequisites behind balance and walking skills, allow elderly to maintain and improve motor performance even in old age (Holviala et al., 2012; Federici \& Lever, 2019). It is therefore essential to change the paradigm from the common stereotype of the inevitability of processes occurring in old age because, in many cases, it is the main culprit for the further deterioration of abilities reduction in functional autonomy (Scatigna et al., 2019). The objectives of a programme aimed at maintaining and improving balance and walking performance include increased body consciousness (proprioception), an improvement in joint mobility, muscle strength, aerobic capacity and 
inter-segmental coordination (Andrieieva et al., 2019). These objectives are complemented by operational proposals aimed at exercising the postural control system by proposing activities of destabilization and stabilization of balance, activities that stimulate the ability to react, combine coordination and motor adaptation, skills indispensable to implement a series of reactions to respond in a timely and effective manner to an event or obstacle that can cause a fall (Coppola et. al, 2015).

The identification of valid and reliable assessment tools, which are able to identify the initial phases of the functional decline of the elderly, allowing the prescription and structuring of a specific physical activity program, represents an important primary prevention and contrast with the establishment of conditions of fragility and loss of independence (Coppola \& Vastola, 2018).

With the recent development of microelectromechanical systems, inertial measurement unit sensors (IMUs) have become widely used in the research of wearable gait analysis due to several factors, such as being easy-to-use and low-cost (Sprager \& Juric, 2015).

The use of inertial systems offers the possibility of performing kinematic analyses that can overcome the spatial-temporal and economic limitations of the traditional approach to the analysis of movement, which can only be carried out within an analysis laboratory movement, using optoelectronic measurement systems, more demanding in terms of costs, time and complexity (Vastola, 2018).

Inertial motion capture systems allow objective evaluation of the main spatial-temporal gait parameters as gait speed, stance percent, swing percent, gait cycle time, stride length, cadence, and step duration (Agostini et al., 2017). Inertial measurement unit (IMUs) for spatiotemporal and kinematic assessments is a major technological advancement in the field of biomechanics and wearable sensors, as they are relatively inexpensive, allow a virtually unlimited number of steps to be evaluated, and provide the ability to evaluate gait and movement disorders outside the constrained environments of the clinic and research laboratory (Washabaugh et al., 2017).

\section{Objective}

Investigate the effects of an adapted physical activity intervention on the main space-time characteristics of elderly walking performance, using inertial motion capture systems.

\section{MATERIALS AND METHODS}

\section{Study design}

This is a pilot study.

\section{Participants}

The research was carried out in two centres for the elderly, one residential centre and one recreation day centre, located in Salerno (southern Italy). The sample consisted of 11 elderly (8 women and 3 men) aged between 70 and 92 years $(82.55 \pm 7.43)$. The selection of the sample was carried out by administering tests that investigated the psycho-physical conditions of the elderly, in order to include in the study those who were in the condition to be able to follow, in complete safety, the APA program. The tests administered for the inclusion of the sample in the study are: Basic Activities of Daily Living (BADL); Instrumental Activities of Daily Living (IADL); Self Report on health, physical activity and fine motor skills; Physical Performance Test; Tinetti Balance and Gait Scale; Short Physical Performance Battery (SPPB); Timed Get Up and Go Test. 


\section{Instruments}

For the analysis of the main spatial-temporal components of the gait in the elderly, a wearable inertial system of motion capture analysis was used. It is composed by a triaxial accelerometer, a magnetic sensor, and a triaxial gyroscope that positioned on L5 allows a functional gait analysis. The system extrapolates from the data acquired all the spatial-temporal gait parameters.

The sensor is positioned at the patient's waist with a special belt which, thanks to its ergonomics, does not influence the execution of the motor gesture. The sample has performed a 7-meter walking test at free speed.

\section{The APA Protocol}

The structured adapted physical activity program was implemented over a period of five months with meetings held twice a week. The adapted physical activity intervention was structured in 50-minute sessions and consisted of specific exercises aimed at increasing muscle strength, flexibility, coordination, static and dynamic balance. The physical activity routine was structured according to the principle of progression, starting from low-intensity exercises until reaching moderately/intense levels of activity. The Protocol consisted of three main phases an initial neuromuscular activation phase, a central phase and a cool-down phase.

The neuromuscular activation phase focuses on walking that was carried out in a progressive manner in terms of time and intensity. If the elder did not show adequate stability, the activity was carried out with the assistance of an operator. At the first four APA intervention, the activity did not include further combined movements. Two weeks after the start of the APA program, arm movements and some other light movements were simultaneously introduced. The exercises introduced at later stages of the APA intervention in this phase (gaits, marching, etc.) aimed at improving the capacity of dynamic balance and increase strength and coordination of the lower limbs in order to promote the prevention of falls.

Central Phase. The middle phase of the APA program included exercises for muscle strengthening, joint mobility, coordination and balance, done almost exclusively by sitting or with the help of the chair as a support for balance. Before starting this phase of the routine, posture while sitting was corrected: back straight, angle between thigh and lower leg at $90^{\circ}$ and legs slightly apart. As in the initial phase, the coordination exercises were proposed in a simple and slow manner but were then progressively integrated with combined and asymmetric exercises. With the same principle of graduality in mind, in the first phase of muscle strengthening exercises a single set of eight executions was proposed and, in later stages, these were extended to two sets of eight. The exercises in this phase are strengthening and joint mobility of the muscular and articular components of the cervical tract, the shoulder girdle, the elbow, the wrist, the column, the pelvis, the hip, the knee and the ankle joint. Cool-down Phase. The cool-down, programmed according to the intensity of the activity performed in the middle phase, aimed at restoring the body to the baseline. Walking combined with breathing exercises is considered particularly favourable to reach this aim with the elderly (Coppola et al., 2015).

\section{Data analysis}

The data were analysed using descriptive statistics in order to trace the relevant parameters. Data are reported as mean \pm standard deviation, as relative or absolute number with the percentage. Statistical analyses were performed using Microsoft Office Excel 2019. 


\section{RESULTS}

The total sample consisted of 11 elderly, 7 women and 4 men, aged between 70 and 92 years. The average age was 82.55 years, with a standard deviation of 7.43 (Table 1). The distribution for sex refers to the entire sample was prevalently constituted by women with a prevalence of $73 \%$ (Table 1). The sample of the recreation day centre was composed of 7 elderly, 3 men and 4 women, with an average age of 79.1 years, while the sample of residential centre consisted of 4 elderly people with an average age of 88.5 years, all female (Table 1).

Table 1. Average age and gender distribution of the sample.

\begin{tabular}{lcccc}
\hline & Men & Women & Average age & SD \\
\hline Day centre & 3 & 4 & 79.14 & \pm 7.31 \\
Residential centre & 0 & 4 & 88.50 & \pm 1.73 \\
Total & 3 & 8 & 82.55 & \pm 7.43 \\
\hline
\end{tabular}

The $45 \%$ of the total sample was constituted by elderly over 85 years old; the other component of the sample was distributed homogeneously in the other age ranges:18\% was in the $70-74$ age range, $18 \%$ was in the $75-79$ age range and the remaining $18 \%$ was in the $80-84$ age range (Table 2 ).

Table 2. Sample distribution by age group with respect, sample structure and total.

\begin{tabular}{|l|ll|l|l|l|}
\hline \multirow{2}{*}{ Day centre } & Age & $70-74$ & $75-79$ & $80-84$ & $85+$ \\
\cline { 2 - 6 } & Elderly & 2 & 2 & 2 & 1 \\
\hline \multirow{2}{*}{ Residential centre } & Age & $70-74$ & $75-79$ & $80-84$ & $85+$ \\
\cline { 2 - 6 } & Elderly & 0 & 0 & 0 & 4 \\
\hline \multirow{2}{*}{ Total centres } & Age & $70-74$ & $75-79$ & $80-84$ & $85+$ \\
\cline { 2 - 6 } & Elderly & 2 & 2 & 2 & 5 \\
\hline
\end{tabular}

Figure 1 shows the average values of the walking speeds of the elderly before and after the APA intervention. Before the APA program, the average speed of the total sample is $1.05 \mathrm{~m} / \mathrm{s}(\mathrm{SD} \pm 0.28)$ and, in particular, in the elderly group of the residential centre is $0.9 \mathrm{~m} / \mathrm{s}( \pm 0.19)$ while that of the elderly group of the day centre is $1.13 \mathrm{~m} / \mathrm{s}(S D \pm 0.30)$. As can be seen from the graph, there is an average increase in speeds in both elderly groups, with a slightly higher increase in the day centre where the increase is $0.09 \mathrm{~m} / \mathrm{s}$ equal to $7 \%$ $(1.22 \mathrm{~m} / \mathrm{s} ; \mathrm{SD} \pm 0.43)$, while in the residential centre an average increase in walking speed of $0.05 \mathrm{~m} / \mathrm{s}$ was recorded, corresponding to $5.5 \%(0.95 \mathrm{~m} / \mathrm{s}$; SD \pm 0.2$)$.

The data relating to the cadence show an average of 110 step/minute (SD \pm 14.19 ) on the total sample of the elderly before the APA intervention and an average value of $107.35 \mathrm{step} / \mathrm{min}$ (SD \pm 17.85 ) after the APA intervention. The cadence of the group of the elderly in the residential centre show an average value of 112.2 step/min (SD \pm 14.32 ) before the intervention of APA and a value of 104.9 step/min (SD \pm 21.17 ) after the APA, with a percentage decrease equivalent to $-7 \%$. The sample of elderly people in the day care centre shows an average cadence value before the APA intervention of $109.4 \mathrm{step} / \mathrm{min}$ (SD \pm 15.15 ) and a value of $108.8 \mathrm{step} / \mathrm{min}$ (SD 17.34) after the APA with a decrease of $-0.05 \%$ (Figure 2). 


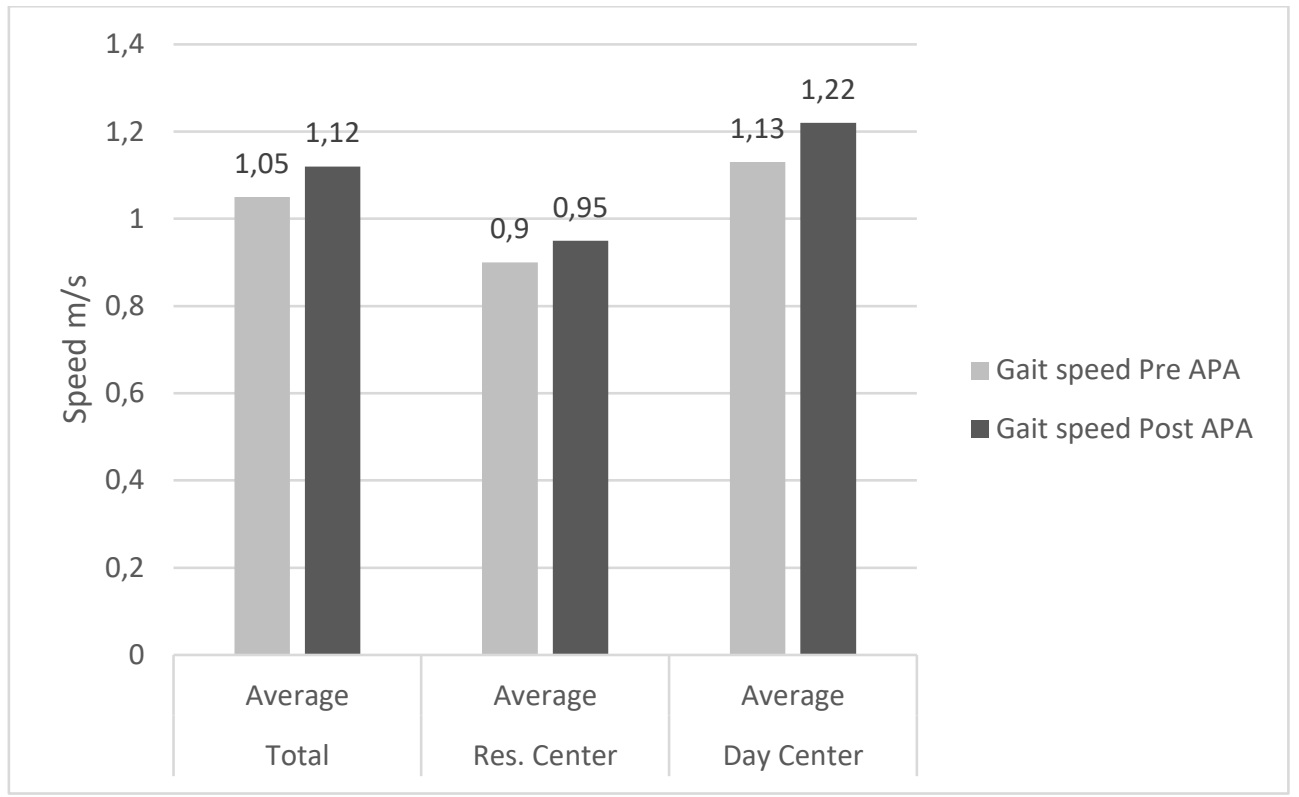

Figure 1. Average of gait speeds of the sample before and after the APA program.

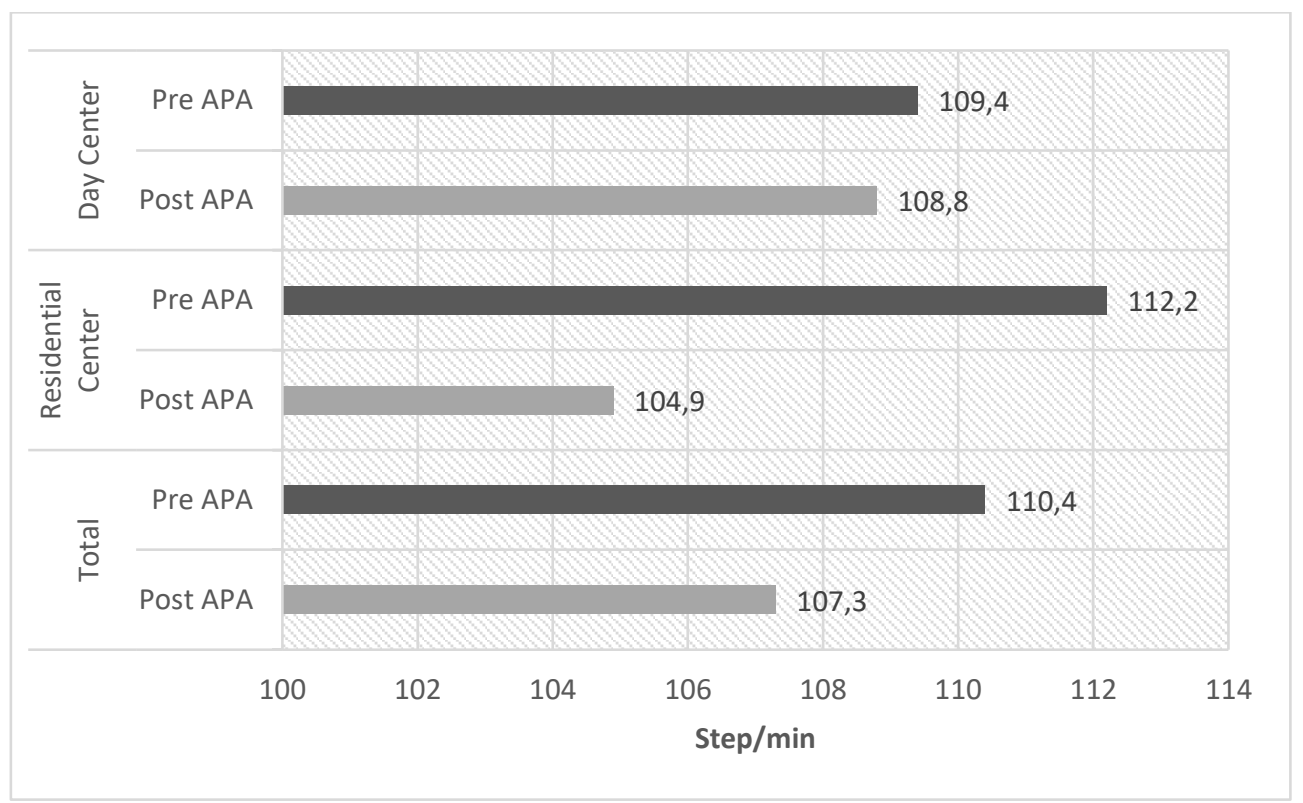

Figure 2. Average of cadence of the sample before and after the APA program.

The data relating to the symmetry index show an initial average value of 0.84 (SD \pm 2.7 ) on the total sample of the elderly before the APA intervention and an average value of 0.85 (SD \pm 1.8 ) after the APA program. Before the APA program the symmetry index of the sample of elderly in the residential centre show an average value of $0.82(S D \pm 2.3$ ) and the sample of day centre an average value of $84(S D \pm 3.6)$. The values after the interventions of APA show an increase of the symmetry in both groups of elderly people with a total average increase of $2.4 \%$, rising to an average value of 86 in the group of the elderly day care centre to a value of 0.84 in the sample of elderly people in the residential centre (Figure 3 ). 


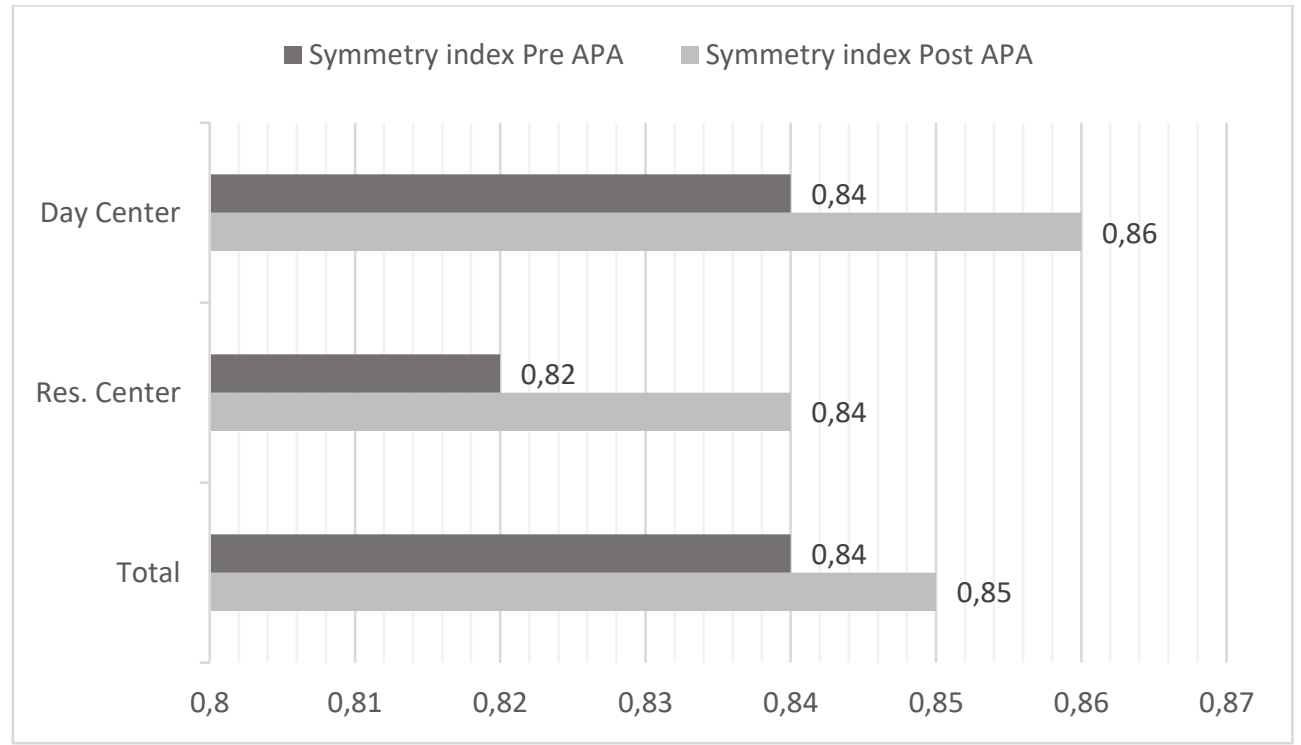

Figure 3. Symmetry index of the sample before and after the APA program.

The stride length data show an average length of $1.12 \mathrm{~m}(\mathrm{SD} \pm 0.2)$ on the total sample of the elderly before the APA intervention and an average value of $1.23 \mathrm{~m}(\mathrm{SD} \pm 0.23)$ after the APA intervention, with an average percentage increase of $9.8 \%$. Specifically, the sample of elderly people from the residential centre shows an average stride length of $0.98 \mathrm{~m}(\mathrm{SD} \pm 0.14)$ before the APA and an average length of $1.10 \mathrm{~m}(\mathrm{SD} \pm 0.17)$ after the intervention of APA $(+11.7 \%)$. The sample of elderly people of the recreational day centre shows an initial average stride length value of $1.2 \mathrm{~m}(\mathrm{SD} \pm 0.18)$ and a post APA average value of $1.31 \mathrm{~m}$ (SD \pm $0.24)(+8 \%)$.Figure 4 presents the data relating to the average length of the stride before and after the APA program, differentiated between the average length of the right and left step in the total of the sample and in the two different centres for the elderly.

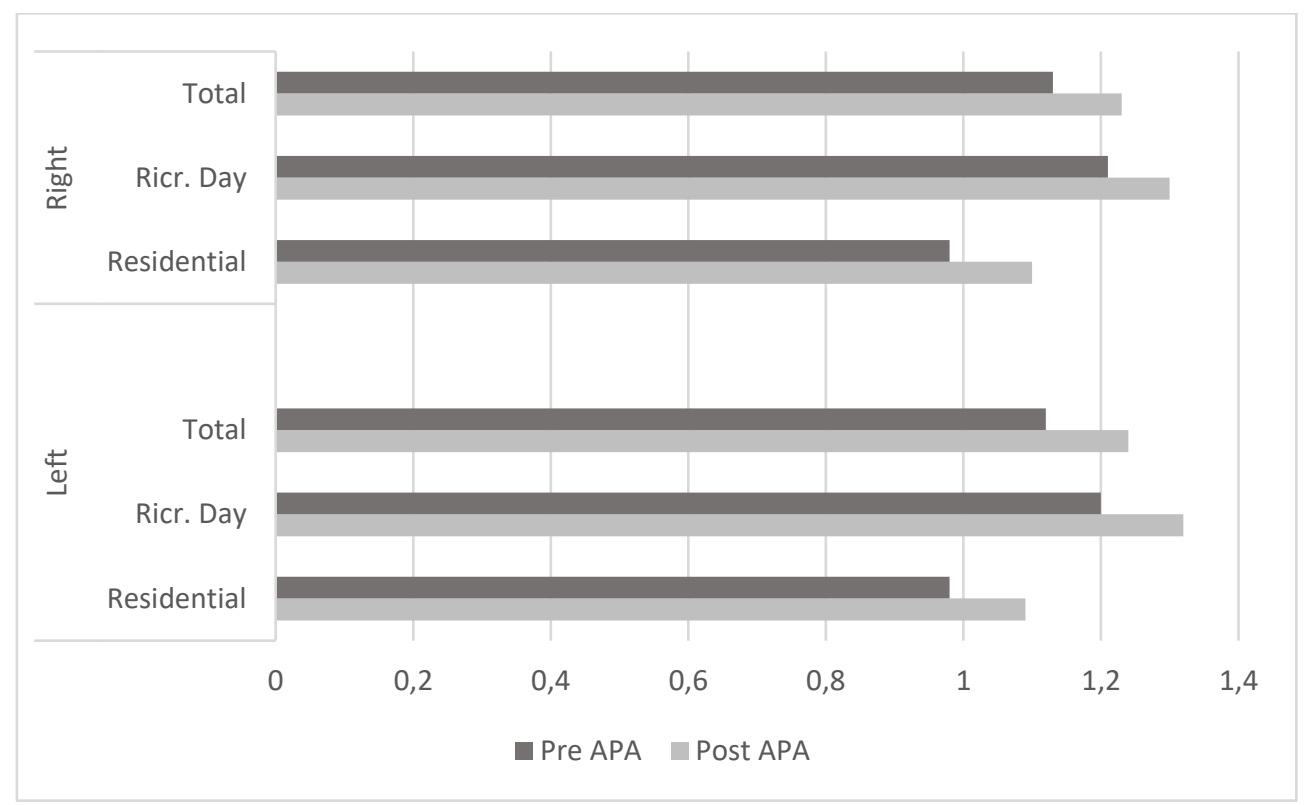

Figure 4. Stride length of the sample before and after the APA program. 
Table 3. Data relating to stride length of the sample before and after the APA program.

\begin{tabular}{|c|c|c|c|c|c|c|c|}
\hline & \multicolumn{2}{|c|}{ Left } & \multicolumn{2}{|c|}{ Right } & \multirow{2}{*}{$\begin{array}{c}\text { \% Difference } \\
\text { Between Left } \\
\text { Tests }\end{array}$} & \multirow{2}{*}{$\begin{array}{c}\text { \% Difference } \\
\text { Between Right } \\
\text { Tests }\end{array}$} \\
\hline & & $\begin{array}{l}\text { Pre } \\
\text { APA }\end{array}$ & $\begin{array}{l}\text { Post } \\
\text { APA }\end{array}$ & $\begin{array}{l}\text { Pre } \\
\text { APA }\end{array}$ & $\begin{array}{l}\text { Post } \\
\text { APA }\end{array}$ & & \\
\hline \multirow{2}{*}{ Residential Centre } & Average & 0.98 & 1.09 & 0.98 & 1.10 & $10 \%$ & $11 \%$ \\
\hline & SD & \pm 0.14 & \pm 0.17 & \pm 0.14 & \pm 0.17 & $\pm 5 \%$ & $\pm 7 \%$ \\
\hline & & & & & & & \\
\hline \multirow{2}{*}{ Day Centre } & Average & 1.20 & 1.32 & 1.21 & 1.30 & $8 \%$ & $6 \%$ \\
\hline & SD & \pm 0.16 & \pm 0.26 & \pm 0.21 & \pm 0.22 & $\pm 8 \%$ & $\pm 6 \%$ \\
\hline \multirow{2}{*}{ Total } & Average & 1.12 & 1.24 & 1.13 & 1.23 & $9 \%$ & $8 \%$ \\
\hline & SD & \pm 0.19 & \pm 0.25 & \pm 0.22 & \pm 0.22 & $\pm 7 \%$ & $\pm 7 \%$ \\
\hline
\end{tabular}

The cycle time of the entire sample of the elderly before the APA intervention is on average $1.09 \mathrm{~s}$ (SD \pm $0.15)$, after the APA it is $1.10 \mathrm{~s}(\mathrm{SD} \pm 0.15)$ equivalent to $0.9 \%$ increase. In particular, the sample of elderly people in the residential centre has an average duration of the cycle time before the APA of $1.02 \mathrm{~s}$ (SD \pm $0.09)$ and $1.05 \mathrm{~s}(\mathrm{SD} \pm 0.07)$ after the intervention of APA. The sample of the elderly in the day centre has an average cycle time duration of $1.12 \mathrm{~s}(\mathrm{SD} \pm 0.16)$ before the APA program and an average value of 1.13 $s(S D \pm 0.17)$ after the intervention APA (Table 4).

Table 4. Cycle time of the sample before and after the APA program.

\begin{tabular}{|l|l|c|c|}
\hline \multicolumn{5}{|c|}{ Cycle Time (s) } \\
\hline \multirow{2}{*}{ Res. Centre } & Average & Pre APA & Post APA \\
\cline { 2 - 4 } & SD & 1.02 & 1.05 \\
\hline \multirow{2}{*}{ Day Centre } & Average & \pm 0.09 & \pm 0.07 \\
\cline { 2 - 4 } & SD & 1.12 & 1.13 \\
\hline \multirow{2}{*}{ Total } & Average & \pm 0.16 & \pm 0.17 \\
\cline { 2 - 4 } & SD & 1.09 & 1.1 \\
\hline
\end{tabular}

Table 5. Stance and swing phase of the sample before and after the APA program.

\begin{tabular}{|l|l|c|c|c|c|}
\hline \multicolumn{2}{|c|}{} & \multicolumn{2}{|c|}{ Stance phase \% } & \multicolumn{2}{c|}{ Swing phase \% } \\
\cline { 3 - 6 } \multicolumn{2}{|c|}{ Res. Centre } & Pre APA & Post APA & Pre APA & Post APA \\
\cline { 2 - 6 } & Average & 64.5 & 63.8 & 35.5 & 36.2 \\
\hline \multirow{2}{*}{ Day Centre } & SD & \pm 1.8 & \pm 1.9 & \pm 1.2 & \pm 1.3 \\
\cline { 2 - 6 } & SDerage & 63.15 & 62.25 & 36.85 & 37.75 \\
\hline \multirow{2}{*}{ Total } & Average & $\mathbf{1 1 . 5}$ & \pm 1.6 & \pm 1.1 & \pm 1.4 \\
\cline { 2 - 6 } & SD & $\mathbf{6 3 . 6 5}$ & 62.82 & 36.35 & 37.18 \\
\hline
\end{tabular}

The values relating to the percentages of the stance phases during the gait cycle of the total sample before the APA intervention are 63.65\% (SD \pm 1.7$)$ and in swing phase are $36.35 \%$ (SD \pm 1.2 ). After the intervention of APA, the average percentage values went respectively to $62.82 \%$ (SD \pm 2.2$)$ in stance phase and to $37.18 \%$ (SD \pm 1.4 ) in swing phase. The sample of elderly people in the residential centre, before the APA, had percentage values in the stance phase of $64.4 \%$ (SD \pm 1.8$)$ and $35.5 \%(S D \pm 1.2)$ in the swing phase. After the APA program the average percentage value in stance phase is $63.8 \%$ (SD \pm 1.9 ) while the swing 
phase value is $36.2 \%$ (SD \pm 1.3 ). The sample of the elderly in the daytime recreation centre has average percentage values before the APA in the stance phase of $63.15 \%$ (SD \pm 1.5 ) and a value of $36.85 \%$ (SD \pm $1.1)$ in the swing phase. After the APA intervention, the average percentage values of the elderly group of the day care centre in stance phase are $62.25 \%(S D \pm 1.6)$ and $37.75 \%(S D \pm 1.4)$ in swing phase (Table $5)$.

Double support phase on the total of the sample before the APA intervention have an average value of 14.2 (SD \pm 4.81 ), after the APA intervention a value of 13.97 (SD \pm 4.95$)$. The sample of elderly people in the residential centre has an average percentage value of double support of 13.1 (SD \pm 3.2 ) and a value of 13 $(S D \pm 4.6)$ after the APA program. The sample of elderly people in the daytime recreation centre has an average percentage of double support before the APA of 12.95 (SD \pm 3.64 ) and a post APA value of 12.25 (SD \pm 4.32 ). The single support phase of the total sample has an average percentage value of $36.66 \%$ (SD \pm 3.27 ) before the implementation of the APA program and a value of $36.96 \%$ (SD \pm 3.68 ). The sample of elderly people in the residential centre has an average value of single support of $36.35 \%$ (SD \pm 1.79 ) before the APA intervention and an average value of $35.63 \%$ (SD \pm 3.43 ) after the APA program. The sample of the elderly in the daytime recreation centre has an average single support value of $36.84 \%$ (SD \pm 3.93 ) before the APA and an average value of $37.72 \%$ (SD \pm 3.71 ) after the APA program (Table 6).

Table 6. Double and single support phase of the sample before and after the APA program.

\begin{tabular}{|l|l|c|c|c|c|}
\hline \multicolumn{2}{|c|}{} & \multicolumn{2}{|c|}{ Double support \% } & \multicolumn{2}{c|}{ Single support \% } \\
\cline { 3 - 6 } \multicolumn{2}{|c|}{} & Pre APA & Post APA & Pre APA & Post APA \\
\hline \multirow{2}{*}{ Res. Centre } & Average & 13.1 & 13 & 36.35 & 35.63 \\
\cline { 2 - 6 } & SD & \pm 3.2 & \pm 4.6 & \pm 1.79 & \pm 3.43 \\
\hline \multirow{2}{*}{ Day Centre } & Average & 12.95 & 12.25 & 36.84 & 37.72 \\
\cline { 2 - 6 } & SD & \pm 3.64 & \pm 4.32 & \pm 3.93 & \pm 3.71 \\
\hline \multirow{2}{*}{ Total } & Average & 14.2 & 13.97 & 36.66 & 36.96 \\
\cline { 2 - 6 } & SD & \pm 4.81 & \pm 4.95 & \pm 3.27 & \pm 3.68 \\
\hline
\end{tabular}

\section{DISCUSSION AND CONCLUSIONS}

The results obtained in this study show a relative improvement in the spatial-temporal parameters of the gait in both elderly groups following the implementation of the structured APA intervention. It was detected an improvement in gait speeds $(+6.2 \%)$, with a slightly higher increase in the sample of elderly day centre where the increase is $0.09 \mathrm{~m} / \mathrm{s}$ equal to $7 \%(1.22 \mathrm{~m} / \mathrm{s}$; SD \pm 0.43$)$, while in the sample of elderly of residential centre an average increase in walking speed of $0.05 \mathrm{~m} / \mathrm{s}$ was recorded, corresponding to $5.5 \%(0.95 \mathrm{~m} / \mathrm{s}$; SD \pm $0.2)$. An average increase of $9.8 \%$ of the stride length was detected $(+0.11 \mathrm{~m})$ with a slightly greater increase in the sample of elderly people in the residential centre $(11.8 \%$ vs $8 \%)$. Contextually as the average increase in speed $(+6.2 \%)$ and step length $(+9.8 \%)$, a consequent decrease in step cadence was recorded $(-2.9 \%)$. The values relating to the percentages of the stance and swing phases show an increase in swing phase of $2.1 \%$ and a decrease of stance of $-1.2 \%$. The data relating to the single and double support, also, show a slight improvement with an average percentage increase of the $0.81 \%$ for the single support phase and an average decrease of $1.6 \%$ for the double support phase. Symmetry index improve similarly in both groups.

Considering that from 65 years the spatial-temporal parameters of the gait undergo a gradual and physiological regression(Whittle, 2014), the results of this pilot study, in relation to the age of the sample that is between 70 and 92 years $(82.55 \pm 7.43)$, could be considered particularly promising. 
In relation to the relatively low number and the relative homogeneity of the sample and the duration of the APA intervention makes this a pilot study. The results are, therefore, rather suggestive than conclusive, and open the field to the need for implement the APA protocol to a larger sample, over a longer period.

In conclusion, based on the results obtained in this study, it is possible to sustain that the inertial motion capture system has proved to be a very effective tool in detecting gait parameters in the elderly and its noninvasive and very versatile features have allowed for evaluation without any difficulty. The protocol of Adapted Physical Activity, despite the absence of a gym and any gym equipment, was successfully and without difficulty implemented in both the elderly residential centre and the daytime recreation centre, demonstrating high feasibility. Finally, this pilot study open the field to the need for experiment this APA protocol and this inertial motion capture system for the gait analysis to a larger sample in order to investigate in more depth the effects of the APA on the main spatial-temporal parameters of the gait of the elderly.

\section{REFERENCES}

Aboutorabi, A., Arazpour, M., Bahramizadeh, M., Hutchins, S. W., \& Fadayevatan, R. (2016). The effect of aging on gait parameters in able-bodied older subjects: a literature review. Aging clinical and experimental research, 28(3), 393-405. https://doi.org/10.1007/s40520-015-0420-6

Agostini, V., Gastaldi, L., Rosso, V., Knaflitz, M., \& Tadano, S. (2017). A wearable magneto-inertial system for gait analysis (H-Gait): Validation on normal weight and overweight/obese young healthy adults. Sensors, 17(10), 2406. https://doi.org/10.3390/s17102406

Andrieieva, O., Hakman, A., Kashuba, V., Vasylenko, M., Patsaliuk, K., Koshura, A., \& Istyniuk, I. (2019). Effects of physical activity on aging processes in elderly persons. Journal of Physical Education and Sport, 19, 1308-1314.

Auvinet, B., Touzard, C., Montestruc, F., Delafond, A., \& Goeb, V. (2017). Gait disorders in the elderly and dual task gait analysis: a new approach for identifying motor phenotypes. Journal of neuroengineering and rehabilitation, 14(1), 7. https://doi.org/10.1186/s12984-017-0218-1

Baker, R., Esquenazi, A., Benedetti, M. G., \& Desloovere, K. (2016). Gait analysis: clinical facts. European journal of physical and rehabilitation medicine, 52(4), 560-574.

Bizovska, L., Svoboda, Z., \& Janura, M. (2015). The possibilities for dynamic stability assessment during gait: A review of the literature. Journal of Physical Education and Sport, 15(3), 490.

Coppola, S. (2018). Adapted Physical Activity in elderly. Analysis of benefits, teaching and assessment tools. In Italian: "L'attività fisica adattata alla terza età. Analisi dei benefici, didattica e strumenti di valutazione". Beau Bassin, Mauritius: Italian Academic Editions, 114-116.

Coppola, S., D'Elia, F., Vastola, R., \& Sibilio, M. (2015). Adapted Physical Activity Interventions among the Elderly: Their Effects on Physical Performance. Journal of Sports Science, 3, 263-271. https://doi.org/10.17265/2332-7839/2015.06.001

Coppola, S. \& Vastola, R. (2018). Adapted Physical Activity and new technologies: a pilot study carried out in Salerno. In Italian: Attività fisica Adatta e nuove tecnologie: uno studio pilota attuato nella provincia di Salerno. Potency of the networks in promoting health. Railegh (USA) Aonia Editor, 231242.

Cruz-Jimenez, M. (2017). Normal changes in gait and mobility problems in the elderly. Physical Medicine and Rehabilitation Clinics, 28(4), 713-725. https://doi.org/10.1016/j.pmr.2017.06.005

De Souto Barreto, P., Morley, J. E., Chodzko-Zajko, W., Pitkala, K. H., Weening-Djiksterhuis, E., Rodriguez-Manas, L., ... \& Izquierdo, M. (2016). Recommendations on physical activity and exercise for older adults living in long-term care facilities: a taskforce report. Journal of the American Medical Directors Association, 17(5), 381-392. https://doi.org/10.1016/j.jamda.2016.01.021 
Fan, Y., Li, Z., Han, S., Lv, C., \& Zhang, B. (2016). The influence of gait speed on the stability of walking among the elderly. Gait \& posture, 47, 31-36. https://doi.org/10.1016/i.gaitpost.2016.02.018

Federici A., Lever R. (2019). Home-fitness: physical exercise and elderly's quality of life. Journal of Physical Education and Sport, 19, 1852 - 1855.

Goble, D. J., Coxon, J. P., Wallace, N., Van Impe, A., \& Swinnen, S. P. (2009). Proprioceptive sensibility in the elderly: degeneration, functional consequences and plastic-adaptive processes. Neuroscience \& Biobehavioral Reviews, 33(3), 271-278. https://doi.org/10.1016/j.neubiorev.2008.08.012

Halaweh, H., Willen, C., Grimby-Ekman, A., \& Svantesson, U. (2015). Physical activity and health-related quality of life among community dwelling elderly. Journal of clinical medicine research, 7(11), 845. https://doi.org/10.14740/jocmr2307w

Hamacher, D., Singh, N. B., Van Dieen, J. H., Heller, M. O., \& Taylor, W. R. (2011). Kinematic measures for assessing gait stability in elderly individuals: a systematic review. Journal of The Royal Society Interface, 8(65), 1682-1698. https://doi.org/10.1098/rsif.2011.0416

Holviala, J., Kraemer, W. J., Sillanpää, E., Karppinen, H., Avela, J., Kauhanen, A., ... \& Häkkinen, K. (2012). Effects of strength, endurance and combined training on muscle strength, walking speed and dynamic balance in aging men. European journal of applied physiology, 112(4), 1335-1347. https://doi.org/10.1007/s00421-011-2089-7

Iwasaki, S., \& Yamasoba, T. (2015). Dizziness and imbalance in the elderly: age-related decline in the vestibular system. Aging and disease, 6(1), 38. https://doi.org/10.14336/ad.2014.0128

Iwata, M., \& Kitamoto, H. (2020). Study on visual acuity curves of the elderly. Japan Architectural Review, 3(1), 135-143. https://doi.org/10.1002/2475-8876.12127

Jahn, K. (2019). The aging vestibular system: dizziness and imbalance in the elderly. In Vestibular Disorders (Vol. 82, pp. 143-149). Karger Publishers. https://doi.org/10.1159/000490283

Jahn, K., Kressig, R. W., Bridenbaugh, S. A., Brandt, T., \& Schniepp, R. (2015). Dizziness and unstable gait in old age: etiology, diagnosis and treatment. Deutsches Ärzteblatt International, 112(23), 387. https://doi.org/10.3238/arztebl.2015.0387

Lee, K., \& Lee, Y. W. (2017). Efficacy of ankle control balance training on postural balance and gait ability in community-dwelling older adults: a single-blinded, randomized clinical trial. Journal of physical therapy science, 29(9), 1590-1595. https://doi.org/10.1589/jpts.29.1590

Miljkovic, N., Lim, J. Y., Miljkovic, I., \& Frontera, W. R. (2015). Aging of skeletal muscle fibers. Annals of rehabilitation medicine, 39(2), 155. https://doi.org/10.5535/arm.2015.39.2.155

Morley, J. E. (2016). Frailty and sarcopenia in elderly. Wiener klinische Wochenschrift, 128(7), 439-445. https://doi.org/10.1007/s00508-016-1087-5

Osoba, M. Y., Rao, A. K., Agrawal, S. K., \& Lalwani, A. K. (2019). Balance and gait in the elderly: A contemporary review. Laryngoscope Investigative Otolaryngology, 4(1), 143-153. https://doi.org/10.1002/lio2.252

Pirker, W., \& Katzenschlager, R. (2017). Gait disorders in adults and the elderly. Wiener Klinische Wochenschrift, 129(3-4), 81-95. https://doi.org/10.1007/s00508-016-1096-4

Scatigna, M., D'Eugenio, S., Cesarini, V., Coppola, L., Lemma, P., Fabiani, L., \& Romano Spica, V. (2019). Physical activity as a key issue for promoting human health on a local and global scale: evidences and perspectives. Ann Ig, 31. 595-613.

Sprager, S., \& Juric, M. B. (2015). Inertial sensor-based gait recognition: A review. Sensors, 15(9), 22089-22127. https://doi.org/10.3390/s150922089

Trueblood, P. R., \& Rubenstein, L. Z. (1991). Assessment of instability and gait in elderly persons: Geriatrics. Comprehensive therapy, 17(8), 20-29.

Vastola, R. (2018). Basic elements of motion analysis. In Italian: Elementi di base di analisi del movimento. Lecce: Pensa Editor, 93-94. 
Warburton, D. E., \& Bredin, S. S. (2016). Reflections on physical activity and health: what should we recommend? Canadian Journal of Cardiology, 32(4), 495-504. https://doi.org/10.1016/.j.cjca.2016.01.024

Washabaugh, E. P., Kalyanaraman, T., Adamczyk, P. G., Claflin, E. S., \& Krishnan, C. (2017). Validity and repeatability of inertial measurement units for measuring gait parameters. Gait \& posture, 55, 87-93. https://doi.org/10.1016/.gaitpost.2017.04.013

Whittle, M. W. (2014). Gait analysis: an introduction. Butterworth-Heinemann, 60-61.

Williams, G. K., \& Vicinanza, D. (2018). Coordination in gait: Demonstration of a spectral approach. Journal of sports sciences, 36(15), 1768-1775. https://doi.org/10.1080/02640414.2017.1416974 